\title{
The correlation potential in density functional theory at the GW-level: spherical atoms
}

\author{
M. Hellgren and U. von Barth \\ Solid State Theory, Institute of Physics, Lund University, Sölvegatan 14A, S-22362 Lund, Sweden
}

(Dated: June 15, 2021)

\begin{abstract}
As part of a project to obtain better optical response functions for nano materials and other systems with strong excitonic effects we here calculate the exchange-correlation (XC) potential of density-functional theory (DFT) at a level of approximation which corresponds to the dynamicallyscreened-exchange or GW approximation. In this process we have designed a new numerical method based on cubic splines which appears to be superior to other techniques previously applied to the "inverse engineering problem" of DFT, i.e., the problem of finding an XC potential from a known particle density. The potentials we obtain do not suffer from unphysical ripple and have, to within a reasonable accuracy, the correct asymptotic tails outside localized systems. The XC potential is an important ingredient in finding the particle-conserving excitation energies in atoms and molecules and our potentials perform better in this regard as compared to the LDA potential, potentials from GGA:s, and a DFT potential based on MP2 theory.
\end{abstract}

PACS numbers: 31.15.Ew, 31.25.-v, 71.15.-m

\section{INTRODUCTION}

The work presented here is part of an ongoing investigation aimed at finding more accurate and computationally efficient ways to calculate optical spectra from materials with strong excitonic effects. Typical examples are most semiconductors, rare-gas crystals, atoms and molecules and nano materials. In these systems the attraction of the excited electron to the hole created in the excitation process is very important and will strongly influence the measured spectra both in the continuum and in the discrete part of the spectra. This so called particlehole effect or vertex correction must be accounted for in the theoretical description in order to have a reasonably accurate, quantitative agreement with the experimental results.

The traditional way to incorporate the particle-hole attraction within many-body perturbation theory is to obtain the optical spectra from solutions to the BetheSalpeter equation for the four-point vertex function. Unfortunately, this is a very demanding procedure already in high symmetry cases like crystalline solids and the method becomes very tough indeed when the symmetry is much lower like, e.g., at surfaces or, even worse, in nano systems. In solids, the two-body wave function describing the particle and the hole is usually expanded in valence- and conduction-band one-electron orbitals leading to huge secular problems.

Nevertheless, the Bethe-Salpeter approach has provided a large number of accurate and useful results in many different systems in spite of the fact that many approximations enter the theory like, e.g., that the actual particle-hole interaction is most often replaced by a statically screened potential. During the past ten years, Time-Dependent Density-Functional Theory (TDDFT) has emerged as a competing and, perhaps, more efficient method for calculating optical absorption spectra including excitonic effects. The central quantity of this ap- proach is the so called exchange-correlation $(\mathrm{XC})$ kernel the knowledge of which, unfortunately, is rather limited. The kernel is the functional derivative with respect to the density of the XC potential which is also a relatively unknown quantity. Due to the pioneering work of several groups within some of the research networks of the European Union ${ }^{1.2}$ it has been shown that the accurate results of the Bethe-Salpeter approach can be reproduced within the framework of TDDFT provided an appropriate $\mathrm{XC}$ kernel is used in the calculation. These results are very encouraging, the drawback being that the method does not provide a recipe for improving the kernel without first improving the underlying approximations within the Bethe-Salpeter approach. But there is a great computational advantage of any approach based on TDDFT as compared to a more standard many-body approach like, e.g., that based on the Bethe-Salpeter equation. The former theories are based on two-point correlation functions whereas the latter on four-point correlation functions.

The simplest approximation to the $\mathrm{XC}$ kernel is the so called adiabatic local-density approximation (ALDA) in which the potential is taken to be the exchangecorrelation part of the chemical potential of the electron gas evaluated at the instantaneous electron density. This simple approach is, however, known to suffer from many ailments. The accurate results of the Bethe-Salpeter approach can, e.g., not be reproduced. The next level of approximation is the exchange-only (EXO) approximation which, to our knowledge, has only been applied once to solids, $\underline{\underline{3}}$ unfortunately, with rather poor results as far as the spectral properties are concerned. The approximation seems, however, to give reasonable excitation energies in localized systems and the total energies calculated from the corresponding response function are rather accurate in all systems,$\underline{4.5}$

Until recently, one of the drawbacks of TDDFT has been the lack of a systematic approach for obtaining successively better approximations to the XC ker- 
nel. Straight-forward many-body perturbation theory (MBPT) can certainly be used to generate approximations to the electronic self energy and the three-point vertex function. But the subsequent conversion into an XC kernel of TDDFT is not at all guaranteed to yield, e.g., a particle conserving density response function. $\frac{6.7}{}$ Particle and current conservation are important properties of physical response functions which should be built-in to their construction. A systematic theory for improved kernels within TDDFT has recently been introduced by us. $\underline{8}$ This theory automatically leads to conserving response functions. It is based on an adaptation to TDDFT of our variational approach to many-body perturbation theory $\underline{9}$ A variational functional of the one-electron Green function is constructed which yields the total energy of the system when evaluated at that Green function which renders the functional stationary. Because of the stationary property of the functional and thus an absence of firstorder errors, accurate energies can be obtained already at rather crude Green functions like, e.g., a non-interacting one 10 The construction of the functional has two basic ingredients: i) a choice of basic functional expression ultimately responsible for the variational quality of the total functional (the size of the second-order corrections to the energies). So far, only two such basic expressions have been considered, one due to Luttinger and Ward 11 and a simpler one due to Klein. $\frac{12}{2}$ The former was shown to have better variational properties than the latter $\stackrel{10}{=}$ However, as we have shown previously,$\frac{8}{\underline{-}}$ the LW functional leads to a response function the calculation of which is beyond our present day capabilities in realistic systems. Therefore, we will here consider only the Klein version of the functional. ii) The choice of $\Phi$ functional which is also a functional of the one-electron Green function $G$. The formal significance of the $\Phi$-functional is that its functional derivative with respect to $G$ yields the electronic self-energy and its physical significance is that it will contain our physical intuition concerning the importance of different physical processes. For instance, in an extended system all the screening diagrams should be included in the $\Phi$-functional and in a system with strong correlation effects it would be appropriate to include some particle-hole and particle-particle ladders. In the present study we will focus on the screening diagrams in the $\Phi$-functional and the resulting electronic self-energy will thus be that of the GW approximation. $\frac{13}{2}$ Some consideration will, however, be given also to the second-order exchange diagram. The variational approach to MBPT is converted into a density-functional theory by a restriction of the variational freedom for the Green function which is taken to be one pertinent to a non-interacting system moving in some local external potential $V(\mathbf{r})$. Due to the Hohenberg-Kohn theorem applied to non-interacting electrons and the one-to-one correspondence between the applied potential and the particle density this restriction immediately turns the variational functional of the Green function into a variational functional of the density. A rewriting of the Klein functional in terms of the particle density recovers the normal form of the total energy of $\mathrm{DFT}^{14}$ in which the so called XC energy becomes the $\Phi$ part of the functional. Functional differentiation with respect to the density yields the $\mathrm{XC}$ potential which is the central object of interest in the present work.

Worked aimed at finding the correct density-functional (DF) potential pertinent to different approximations started a long time ago. Even before the advent of DFT, Sharp and Horton $\frac{15}{}$ proposed a method for finding a local potential which would accurately reproduce the total energies and densities of atoms within the Hartree-Fock approximation. This work is, in todays language, best referred to as the first appearance of the Exchange-Only (EXO) approximation or the Exact-Exchange (EXX) method. This method, sometimes also referred to as an optimized potential method (OPM) was later used by Talman and Shadwick ${ }^{16}$ for doing calculations on many atoms. In 1982, one of us made use of the HohenbergKohn theorem to find that local potential which exactly reproduces the Hartree-Fock densities of several atoms $\frac{17}{17}$ These calculations are not equivalent to the EXO but the results are, numerically, very close indeed. Shortly afterwards, this numerical fitting procedure was generalized to include also all correlation effects thus producing 18 the first exact DF XC potentials for several atoms. As a matter of fact, the exact XC potential for the Helium atom was known already at that time through the work of Smith, Jagannathan and Handler $\underline{19}$ Aryasetiawan and Stott ${ }^{20,21}$ also found the exact XC potential of DFT for some smaller atoms using a presumably more accurate approach which had the additional advantage of offering insight into the so called v-representability problem of DFT. In solids, early progress toward an exact DF XC potential was made by Godby et al $\stackrel{22,23}{2}$ who actually constructed their XC potential as a solution to the Linearized Sham-Schlüter equation (LSS) ${ }^{23}$ The self-energy of their choice was again that of the GW approximation (GWA). Through the formal proofs of the present work we now know that their potential was in fact the full RPA $\mathrm{XC}$ potential of DFT for the semiconductors they studied. Consequently, the work of Godby et al. was almost identical in spirit to that of the present work, albeit in solids $\underline{24}$

From the middle of the eighties the number of publications describing work aimed at finding improved $\mathrm{XC}$ potentials for DF calculations increased rapidly - both with regard to approximations for use in practical calculations and with regard to a fundamental understanding of the behavior of the potentials in exact cases and in model systems. The most accurate XC potentials produced thus far are probably those published by Umrigar and Gonze: ${ }^{25}$

In the present work we concentrate on the $\mathrm{XC}$ potential at the GW level which we here prove to be identical to that which minimizes the standard expression for the total energy within the Random Phase Approximation (RPA). Our main motivation is an interest in density and current response functions beyond the EXO and the 
$\mathrm{XC}$ potentials then constitutes one of the basic ingredients. Through the work of, e.g., Petersilka et al ${ }^{26}$ it has, however, long been realized that the accuracy of the XC potentials is crucial for obtaining accurate excitation energies from TDDFT. Thus, it is certainly of interest to see how well our GW-based potentials perform in this context. In addition, these potentials provide a good testing ground for our new numerical approach based on splines as basis functions for electronic structure calculations in atoms, molecules, and solids.

The paper is organized as follows. In Sec. II we shortly present the formal framework based on the variational approach to MBPT. In Sec. III we present our new numerical approach and discuss its advantages and shortcomings. Numerical results for several spherically symmetric atoms are given in Sec. IV. We discuss the behavior of the GW/RPA potentials and compare their performance to that of potentials of other approximations, like, e.g., the EXX and the MP2. We also calculate particleconserving excitation energies using our calculated potentials in conjunction with the approximate $\mathrm{XC}$ kernel of PGG $\stackrel{\underline{4}}{=}$ Finally, in Sec. V, we draw our conclusions as well as advertise our forthcoming work on response functions.

\section{CONSERVING APPROXIMATIONS WITHIN TDDFT}

Physical observables of a system of interacting electrons can be calculated within MBPT, where the central quantity is the one-particle propagator, or the Green function $G$. The latter has a diagrammatic expansion in powers of the Coulomb interaction which, in extended systems, always must be carried to infinite order. Guided by physical intuition, approximations for $G$ can be constructed by including only a selected set of diagrams, appropriate to the system studied. The expansion of $G$ can be written in terms of Dyson's equation,

$$
G=G_{\mathrm{H}}+G_{\mathrm{H}} \Sigma G,
$$

where $G_{\mathrm{H}}$ is the Hartree Green function and $\Sigma$ is the self energy which contains all the many-body effects above the Hartree level. The Hartree Green function $G_{\mathrm{H}}$ is the one-electron propagator of non-interacting electrons moving in the total potential consisting of the external potential $w$ and the Hartree potential, i.e., the Coulomb potential from the total electronic charge density.

Within MBPT, also the density response function $\chi$ has an expansion in powers of the Coulomb interaction. Choosing only a subset of diagrams, albeit an infinite subset, results in an approximate response function which only by pure chance will obey important conservation laws like, e.g., particle number, momentum and energy conservation. A scheme to construct approximations within the framework of MBPT which are conserving was first proposed by Kadanoff and Baym. 27,28 They made use of a functional $\Phi[G]$ with the property that its functional derivative with respect to $G$ is the self energy

$$
\Sigma=\frac{\delta \Phi}{\delta G}
$$

As a consequence, the self energy $\Sigma$ will be a functional of the interacting Green function $G$. The response to external perturbations of a system treated within such an approximation will involve the derivative of the self energy with respect to $G$ and consequently a symmetric second derivative of $\Phi$ with respect to $G$. It can be shown that this symmetry is a sufficient condition for the conserving properties of the resulting response function. Approximations generated from this scheme are called $\Phi$-derivable.

About the same time, Klein $\frac{12}{2}$ constructed a variational functional of $G$ composed of the $\Phi$-functional and some additional terms,

$$
i Y_{\mathrm{K}}[G]=\Phi[G]-\operatorname{Tr}\left\{G G_{\mathrm{H}}^{-1}-1+\ln \left(-G^{-1}\right)\right\}-i U_{\mathrm{o}}[G] .
$$

Here, $U_{\mathrm{o}}$ is the classical Coulomb interaction energy between the electrons given by $U_{\mathrm{o}}=\frac{1}{2} \int n v n$. When this Klein functional is varied with respect to $G$ it is seen to be stationary when $G$ obeys Dyson's equation, Eq. (1). Moreover, at the stationary point the functional takes the value of the ground state energy of the system. The functional is general and applies to any system; the reference to the particular system is contained in the Hartree Green function, $G_{\mathrm{H}}$. Other functionals of $G$ and $\Phi$ have been designed like, e.g., the LW functional ${ }^{11}$ or the ABL functional. $\stackrel{9}{~ T h e s e ~ f u n c t i o n a l s ~ h a v e ~ d i f f e r e n t ~ a n d ~ g e n-~}$ erally better variational properties as compared to the Klein functional and we refer the reader to Ref. 10 for a more comprehensive discussion. In this work, however, we will focus our attention on the Klein functional.

Starting from the Klein functional, we can restrict the variational freedom of the Green function to noninteracting ones, $G_{s}$, generated by a local multiplicative potential, $V$. The Klein functional then becomes a functional of that potential. From the Hohenberg-Kohn theorem there is a one-to-one mapping between the particle density and the potential which turns the Klein functional into a functional of the density and our theory into a time dependent density functional theory. The simplicity of a non-interacting Green function allows us to convert the Klein functional into the form,

$$
Y_{\mathrm{K}}[V]=-i \Phi\left[G_{s}\right]+T_{s}[n]+\int w n+U_{\mathrm{o}},
$$

where $T_{s}$ is the kinetic energy of non-interacting electrons and $w$ is the external potential. It is now clear that $-i \Phi$ plays the role of the XC energy, $E_{\mathrm{xc}} \stackrel{14}{\underline{14}}$ Varying this form with respect to the potential $V$ we find it to be stationary when the potential is given by

$$
V=w+V_{\mathrm{H}}+v_{\mathrm{xc}},
$$




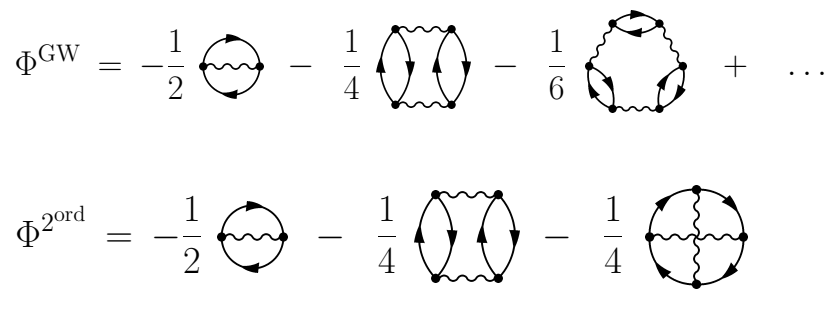

FIG. 1: Diagrammatic representation of the $\Phi$-functional in the two approximations studied in this paper.

where $V_{\mathrm{H}}=\int n v$ is the Hartree potential and where $v_{\mathrm{xc}}$ obeys

$$
\int i \chi_{s}(1,2) v_{\mathrm{xc}}(2) d 2=\int \Sigma(2,3) G_{s}(3,1) G_{s}(1,2) d(23) .
$$

This is the well known so-called linearized Sham-Schlüter (LSS) equation ${ }^{23}$ which here is seen to follow from a variational principle rather than being just the first iteration of an infinite number of iterations leading to the solution to the full Sham-Schlüter equation $\stackrel{29,30}{=}$ We here remark that there is also a self-consistency procedure involved in solving the LSS because the non-interacting so-called Kohn-Sham (KS) response function $\chi_{s}$ as well as the self energy $\Sigma$ are both expressed in orbitals obtained from solving a one-electron Schrödinger equation in which the unknown XC potential $v_{\mathrm{xc}}$ is part of the local potential. As pointed out by Casida, it is also worth noting that $v_{\mathrm{xc}}$ obtained in this way can be seen as the best local approximation to the self energy in a variational sense 31

The conserving properties, and particle conservation in particular, are important when calculating response functions from TDDFT. Within TDDFT, the interacting density response function $\chi$ can be shown to be given by ${ }^{32}$

$$
\chi=\chi_{s}+\chi_{s}\left[v+f_{\mathrm{xc}}\right] \chi,
$$

where

$$
f_{\mathrm{xc}}=\frac{\delta v_{\mathrm{xc}}}{\delta n} .
$$

A further variation of Eq. 5 with respect to the density gives us an equation for the two-point XC kernel, $f_{\mathrm{xc}} \stackrel{\text { 을 }}{=}$ And, because of the underlying $\Phi$-derivability of the theory the resulting response function obeys, e.g., particle conservation which, in the linear response regime, amounts to the $f$-sum rule. Another important property which also follows from the variational and conserving character of this theory is the well known Virial Theorem which can be used as a stringent test of the accuracy of the calculation of the total energy. In Appendix A the reader can find an explicit derivation of that theorem in the context of the present theory.

\section{A. The GW approximation}

In this work we are interested in investigating the DF approximation resulting from choosing $\Phi$ in the GWA, see Fig. 1. This approximation corresponds to a summation of all ring diagrams, where the propagators are KS Green functions,

$$
\Phi^{\mathrm{GW}}=\frac{1}{2} \operatorname{Tr}\left\{\ln \left(1+i v G_{s} G_{s}\right)\right\} .
$$

Inserting $\Phi^{\mathrm{GW}}$ into the Klein functional results in an energy expression corresponding to the well known random phase approximation (RPA) for the total energy. Consequently, the total energy within the RPA is a variational expression and the stationary point has been proven to be a minimum. ${ }^{33}$ Its minimization with respect to the density yields the ground-state energy and density as well as the corresponding $\mathrm{XC}$ potential $v_{\mathrm{xc}}$.

Taking the functional derivative of $\Phi$ in Eq. (7) we obtain the self energy,

$$
\Sigma(1,2)=i G_{s}(1,2) W(1,2),
$$

where the effective interaction $W$ is given by

$$
W=\left[1-v \chi_{s}\right]^{-1} v,
$$

and $\chi_{s}$ is the KS non-interacting polarization propagator. The LSS-equation can then be split into two terms and written symbolically as

$$
\int \chi_{s} v_{\mathrm{xc}}=\int i G_{s}\left[v+v \chi^{\mathrm{RPA}} v\right] G_{s} .
$$

Keeping only the first term, the Hartree-Fock term, results in what is known as the exact exchange (EXX) approximation or, sometimes, the exchange only (EXO) approximation and has been discussed earlier by several

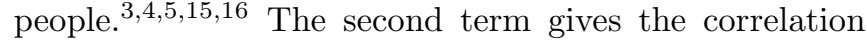
part of the potential and is expressed in terms of the interacting polarization propagator $\chi^{\mathrm{RPA}}$, which, within the ring approximation or the RPA, is given by

$$
\chi^{\mathrm{RPA}}=\chi_{s}+\chi_{s} v \chi^{\mathrm{RPA}} .
$$

In the following we will denote the self-consistent $\mathrm{XC}$ potential corresponding to the GW-level of the Klein functional by $v_{\mathrm{xc}}^{\mathrm{RPA}}$. This potential is more commonly referred to as the XC potential of the RPA.

\section{B. The second order approximation}

In the previous section a summation of $\Phi$-diagrams up to infinite order in the Coulomb interaction is carried out. A conserving approximation does, however, not require an infinite set of $\Phi$-diagrams. In the conserving second order approximation, also known as the Born approximation, all diagrams which are at most second order in 
the Coulomb interaction and only those diagrams are included in the $\Phi$-functional, see Fig 1. Except from the first order Fock diagram there are then, in total, two more diagrams to be considered. One is the first screening diagram, also included in the GWA. The second is the first vertex diagram also referred to as the second order exchange diagram and it is not included in the GWA.

When considering only non-interacting KS Green functions and choosing $\Phi$ in the second order approximation the resulting energy expression is easily seen to be equivalent to the second order energy expression obtained in Møller -Plesset perturbation theory (MP2). This approximation is common in quantum chemistry where the inserted orbitals are those of the Hartree-Fock approximation. In our variational approach also the MP2 expression for the total energy can be minimized with respect to the density thus yielding a minimizing local $\mathrm{XC}$ potential. This potential was recently calculated by Jiang and Engel. ${ }^{34}$ For the purpose of testing our new numerical approach described in the next section, we have repeated their calculation. In accordance with the notation of their paper we will denote that correlation potential $v_{\mathrm{c}}^{\mathrm{MP} 2}$.

\section{NUMERICAL APPROACH}

The solution to the linearized Sham-Schlüter equation is complicated by the singularities of the kernel $\chi_{s}$ giving rise to numerical instabilities. Also, $\chi_{s}$ contains an infinite number of unoccupied states leading to integrals over the continuum. When, like here, a finite basis is used these integrals turn into discrete sums. Previous atomic and molecular calculations have lead to both unphysical oscillations and to an incorrect asymptotic behavior of the potential, as has been discussed by many authors $34,35,36,37,38$ In an attempt to avoid these difficulties we have designed a new basis set consisting of cubic splines.

\section{A. Cubic splines as radial basis functions}

We start by distributing a set of five nodes, not necessarily equidistant, along the radial axis,

$$
R=\left\{r_{k}: k=0, \ldots, 4 ; r_{k}<r_{k+1} ; r_{k} \in \mathbb{R}\right\}
$$

From these nodes we can form a localized, piecewise third-order polynomial function $S$, in the following way:

1. Cubic polynomials $P_{k}=a_{k} r^{3}+b_{k} r^{2}+c_{k} r+d_{k}$, are defined on the four intervals $I_{k}=\left[r_{k-1}, r_{k}\right]$, $k=1, \ldots, 4$. In each of these $S(r)=P_{k}(r)$.

2. When $r \leq r_{0}$ and $r \geq r_{4}$, the function $S$, is zero.

3. The function $S$, is required to be continuous and to have a continuous first and second derivative on the whole real axis. This means that for $k=1, \ldots, 3$ :

$$
\begin{aligned}
& P_{k}\left(r_{k}\right)=P_{k+1}\left(r_{k}\right) \\
& P_{k}^{\prime}\left(r_{k}\right)=P_{k+1}^{\prime}\left(r_{k}\right) \\
& P_{k}^{\prime \prime}\left(r_{k}\right)=P_{k+1}^{\prime \prime}\left(r_{k}\right),
\end{aligned}
$$

and at the end-points:

$$
\begin{array}{rlrl}
P_{1}\left(r_{0}\right) & =0, & P_{4}\left(r_{4}\right)=0 \\
P_{1}^{\prime}\left(r_{0}\right)=0, & P_{4}^{\prime}\left(r_{4}\right)=0 \\
P_{1}^{\prime \prime}\left(r_{0}\right)=0, & P_{4}^{\prime \prime}\left(r_{4}\right)=0 .
\end{array}
$$

Functions designed in this manner are in the numerical literature called cubic splines 39 From the way the spline above is constructed there are sixteen unknown coefficients of the four cubic polynomials and fifteen matching conditions. The coefficients can then be determined up to a common factor. By fixing this factor the spline is uniquely defined on the given set $R$.

For the purpose of building up a basis set, we distribute a set of mesh points $M=\left\{r_{k}: k=0, \ldots, N+3 ; r_{k}<\right.$ $\left.r_{k+1} ; r_{k} \in \mathbb{R}\right\}$, along the radial axis. From the set $M$ we can define the subsets $R_{i}=\left\{r_{k}: k=i, \ldots, i+4 ; r_{k} \in R\right\}$ and on every subset $R_{i}$ we can define a spline $S_{i}$. This generates a basis set of $N$ splines with a distribution in space determined by the choice of mesh. This mesh should certainly be chosen to suit the physical problem at hand. One of the first computer codes for atomic calculations was constructed by Herman and Skillman 40 who chose radial mesh points with a separation increasing quadratically with the distance from the nucleus - a so called cubic mesh. In later years an exponential mesh became more common but the idea is similar. It is important to stack points close to the nucleus in order to account for the rapid oscillations produced by the strong nuclear potential while a much coarser mesh is sufficient in the outskirts of the atom where the wave functions decay exponentially. The exponential mesh is extreme in the sense that one obtains a very accurate description close to the nucleus whereas that mesh gives a poor description some distance away from the atom. This is fine if only occupied states of single atoms are considered. MBPT requires also a reasonable description of the excited one-electron orbitals for which the exponential mesh is inadequate. It would also be less appropriate if one should like to add another atom some distance away to form a molecule. Thus, we have here settled on a cubic mesh and have chosen our mesh points according to $r_{k}=[h(k-3)]^{3}, k=0, \ldots, N+3$ where the 'spacing' $h$ is determined by the relation $r_{\max }=[h(N-3)]^{3}$. This choice is further supported by convergence tests carried out by Stankovski. $\stackrel{41}{=}$ Consequently, our entire numerical procedure has two basic parameters, the number of splines $N$ and the maximum radius $r_{\max }$ outside which no physics is of interest to us. We are, e.g., not interested in highly excited states or in scattering problems. We are interested in low lying excitations or in higher excitations only to the extent that they indirectly affect 
the low-energy excitations. Of course, the convergence of our results with respect to both these numerical parameters have been thoroughly checked. But we find it essential to stress that, due to the completeness of the splines, the results must converge toward the exact results for the chosen physical approximation when $N$ and $r_{\max }$ are made arbitrarily large. Thus, there is no need to discuss the dependence of the results on the quality of the chosen basis set and on the choice of particular exponents of Gaussians or of Slater functions.

The KS equation is a second order differential equation the solutions of which are required to have a continuous first derivative. A potential with no discontinuities in the form of steps gives rise to solutions with a second derivative which is also continuous. Consequently, our cubic splines fulfill the basic requirements for radial basis functions and they have the additional advantage of constraining the potential to be continuous, which is a property reflecting our prejudices concerning a proper $\mathrm{XC}$ potential.

Before ending this subsection, we would like to mention a further important numerical consequence of using splines as basis functions. Every single spline does only overlap with its three closest neighbors on both sides. Consequently, the matrices of the corresponding secular problem are band matrices for which there exist numerous efficient diagonalization algorithms.

\section{B. Re-expansion procedures}

The general practical procedure followed here in order to find the XC potential pertinent to the GW approximation is as follows. A starting potential like that of the simple LDA is used to generate a KS non-interacting Green function $G_{s}$. From this Green function we easily obtain the KS non-interacting density response function $\chi_{s}$. The time-ordered version of $\chi_{s}$ is

$$
\chi_{s}\left(\mathbf{r}, \mathbf{r}^{\prime}, \omega\right)=\sum_{q} \frac{f_{q}(\mathbf{r}) f_{q}^{*}\left(\mathbf{r}^{\prime}\right)}{\omega^{2}-\left(\omega_{q}-i \eta\right)^{2}}
$$

where $q=(k, \mu)$ is a particle-hole index, $\omega$ is the frequency, $\omega_{q}=\epsilon_{\mu}-\epsilon_{k}$ is a particle-hole excitation energy, and $f_{q}$ is an 'excitation amplitude', i.e., a product of the occupied KS orbital $\varphi_{k}$ and the unoccupied KS orbital $\varphi_{\mu}$. The fact that the KS response function $\chi_{s}$ is diagonal in this 'excitation basis' allows for a very simple and efficient way of solving for the full RPA density response function $\chi^{\mathrm{RPA}}$ of Eq. (11).

Consequently, a substantial part of the numerical effort must be spent on finding an efficient an accurate way of representing the product of two one-electron orbitals. Just solving the ordinary KS equations presents a similar problem. At every step toward self-consistency the electron density, being the sum of the squares of the occupied orbitals, must be re-calculated. In a numerical approach based on localized orbitals an obvious choice of basis for the products of the wave functions would be the product of the basis functions. With $N$ basis functions, this means that one would use $N^{2}$ basis functions for the product functions meaning, e.g., that the matrix describing the response function $\chi_{s}$ would be $N^{2} \times N^{2}$. This is clearly an unnecessary effort. For instance, people using an approach based on LMTO:s would use products of LMTO:s for describing $\chi_{s}$ but would only use a subset of the $N^{2}$ products of LMTO:s. The actual product basis functions included can, e.g., be determined numerically by measuring the degree of variational freedom gained by adding one extra product basis function $\underline{42}$ Unfortunately, in this way, one may be able to reduce the number basis functions for the products by a factor of two or three which is not a very large gain if $N$ is large. In the case of methods based on plane waves the situation is slightly better. If the accuracy of the wave functions are considered enough by including all plane waves up to a chosen momentum cut-off, quantities like the density and the excitation functions will contain, on the average, eight times as many plane waves as the wave functions.

In our case, using the $N$ cubic splines as basis functions, our excitation functions would be a sum of polynomials of degree six. But if it is sufficient to describe the wave functions in terms of $N$ polynomials of the third degree, intuition suggests that the same should be true also for the density and the excitation functions. We thus reexpand products of wave functions in terms of the same set of cubic splines as used for the wave functions. The accuracy of this intuition has to be verified numerically. We have found that, in the case of a fixed atomic potential - i.e. no charge density or self-consistency is involved - the accuracy of the KS eigenvalues increases by an order of magnitude and the relative error decreases to $10^{-5}$ when increasing the number of splines from twenty to thirty. However, including also errors arising from the re-expansion of the self-consistent density in terms of the same number of cubic splines as used for the wave functions this error increases by a factor of three at thirty splines. By a rather minor increase in the number of splines - as compared to eight times the number of plane waves or the number of localized basis functions squared, we regain the full accuracy $\left(10^{-5}\right)$ at just below forty cubic splines. From this we conclude that the cubic splines constitute a superior basis set for many-body calculations involving two-point correlation functions.

The so obtained functions $\chi^{\mathrm{RPA}}$ and $G_{s}$ are finally used to calculate the screened interaction $W$ and the self-energy $\Sigma$ of the GWA. And then the LSS, Eq. (5) is solved for the $\mathrm{XC}$ potential $v_{\mathrm{xc}}$ by expanding it too in our cubic splines and inverting $\chi_{s}$ expressed as a matrix in cubic splines. As discussed above this matrix is singular. The physical reason for this is that the response of a constant potential is zero. Inverting $\chi_{s}$ is thus not a unique operation. This difficulty can be circumvented by adding the constraint:

$$
\lim _{r \rightarrow \infty} v_{\mathrm{xc}}(r)=0 .
$$


Mathematically this means to invert the matrix $\chi_{s}$ in the subspace orthogonal to that defined by the zero eigenvalue of $\chi_{s}$.

In all our calculations the EXX potential was first calculated and then used as a starting guess. The convergence criterion in our calculations was set to $\mid n^{(k)}(r)-$ $n^{(k-1)}(r) \mid \leq 10^{-5}$, and was reached in a few iterations for all spherical atoms up to Ar.

\section{RESULTS FOR SPHERICAL ATOMS}

In the present paper we present results for the spherical atoms $\mathrm{He}, \mathrm{Be}, \mathrm{Ne}, \mathrm{Mg}$ and $\mathrm{Ar}$, see Figs. 2,4. In the cases of $\mathrm{He}, \mathrm{Be}$ and Ne we compare our results to existing exact density functional potentials $2 \frac{25}{2}$

\section{A. The RPA correlation potential}

The correlation potentials in this work are defined as the difference between the self-consistent XC potentials calculated within the RPA, or MP2, and that of the EXX,

$$
v_{\mathrm{c}}(r) \equiv v_{\mathrm{xc}}\left[n_{\mathrm{xc}}\right](r)-v_{\mathrm{x}}\left[n_{\mathrm{x}}\right](r) .
$$

Note that the correlation potential is defined as the difference between potentials calculated at two different densities. For a more detailed discussion of this point we refer to Sec. IV C.

In Fig. 2 the RPA and MP2 correlation potentials for $\mathrm{He}, \mathrm{Be}$ and $\mathrm{Ne}$ are presented and compared to the exact correlation potentials $\underset{25}{2}$ The characteristic shell oscillations inherent in the exact potential can not be reproduced by potentials depending explicitly on the density. Indeed, both LDA- and gradient corrected potentials lack the correct shell oscillations (see e.g. Ref. 34). The potential $v_{c}^{\mathrm{RPA}}$, however, which is an implicit density functional through the dependence on the KS orbitals and eigenvalues, can be seen to reproduce these oscillations very well and this is also the case for the potential of MP2. Except at the origin, the amplitudes of the oscillations of $v_{c}^{\mathrm{RPA}}$ are much closer to those of the exact potential as compared to the case of $v_{c}^{\mathrm{MP} 2}$. At the origin the RPA potential appears to deviate the most from the exact potential by being too attractive for all atoms. It should then be remembered that the values of the potential in this region is expected to be of less importance due to the strong singular Coulomb potential from the nucleus. It is tempting to interpret the superiority of the RPA correlation potential to that of the MP2 in the outer parts of the atoms as an increased importance of screening in this region. Similarly one might guess that short range correlations are more important in the interior of the atoms leading to a better performance of the MP2 potential in this region.

In order to demonstrate the effect of correlations on the total XC potential we compare the RPA version of $v_{\mathrm{xc}}$
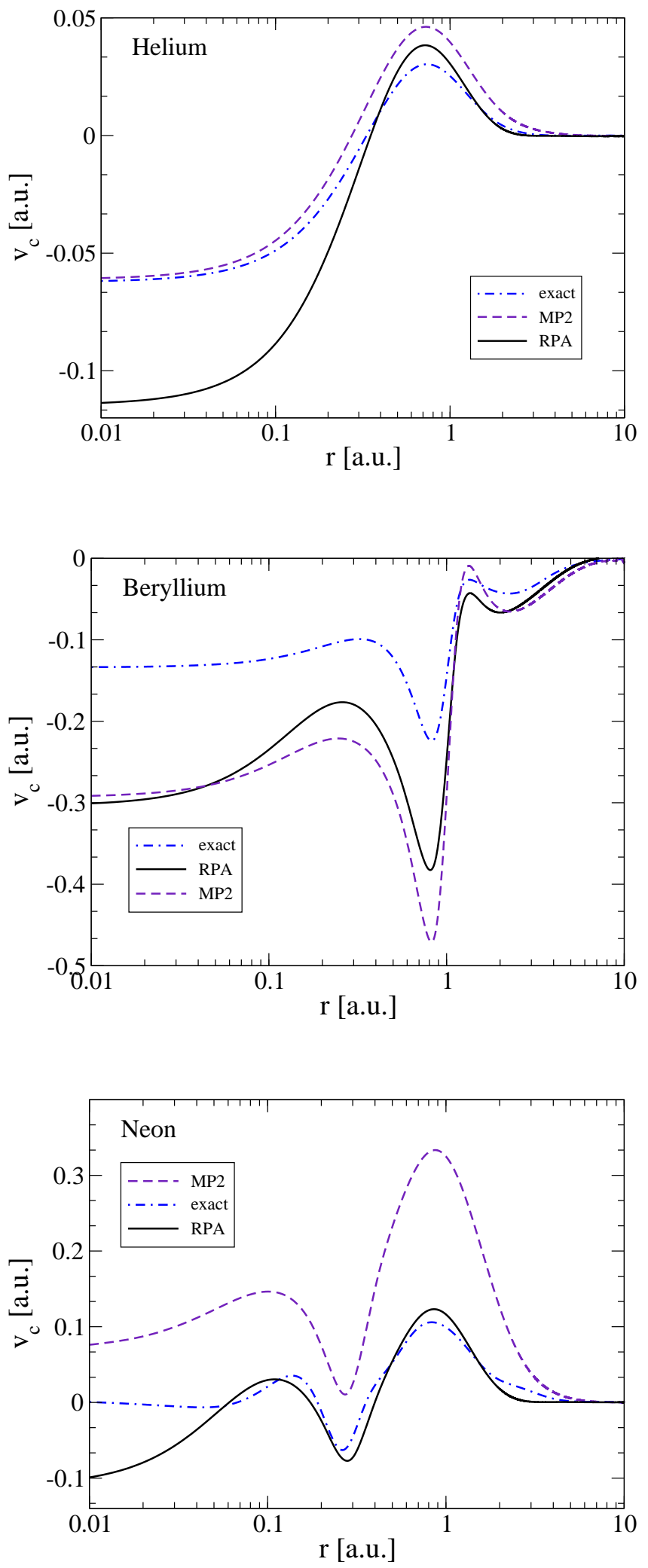

FIG. 2: Self-consistent correlation potentials for $\mathrm{He}, \mathrm{Be}$, and Ne. The RPA-potential is compared to the MP2-potential and to the exact potential. For Be no self-consistent MP2potential can be obtained. Instead, the potential was evaluated at the EXX density. For a further discussion see Ref. 34 

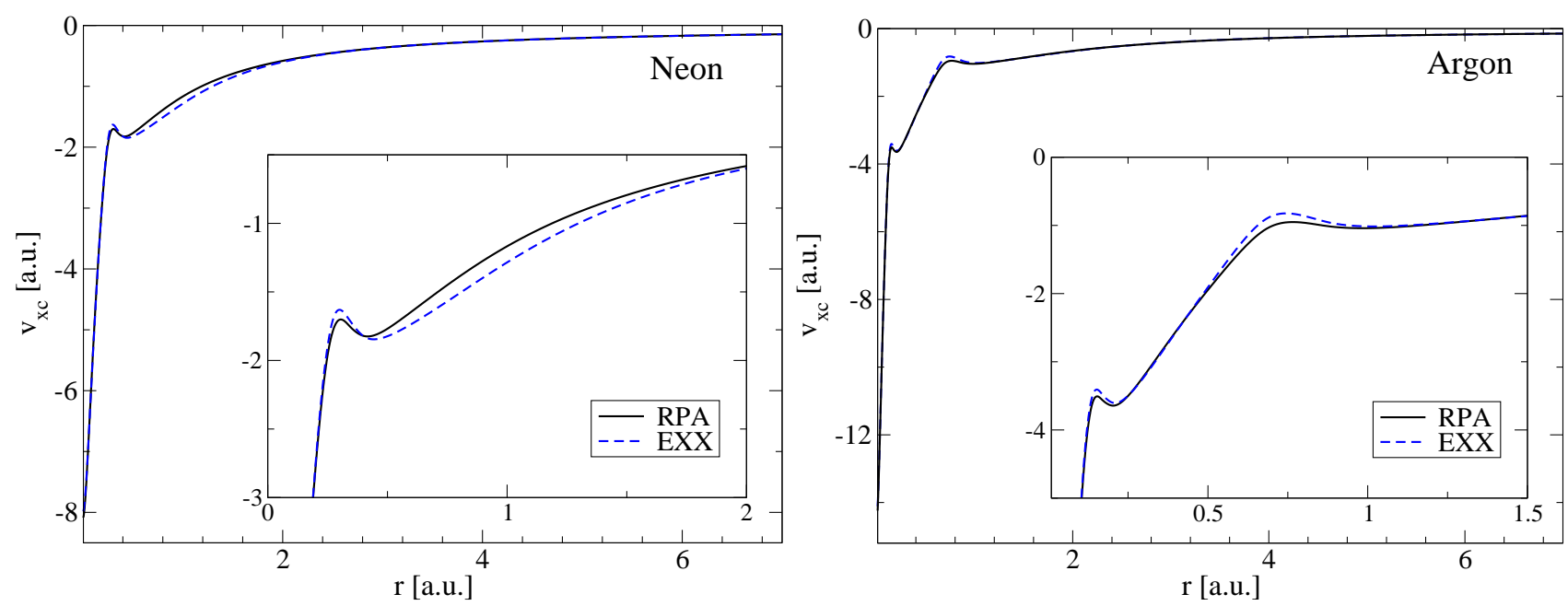

FIG. 3: The total XC potential $v_{\mathrm{xc}}^{\mathrm{RPA}}$ of Ne and Ar compared to $v_{\mathrm{x}}$. The effect of correlations is seen to be relatively small and, as expected, the shell oscillations are damped by the inclusion of correlation effects.

to the potential of $v_{\mathrm{x}}$ of EXX in Fig. 3 (for Ne and Ar). As expected, the shell oscillations are damped by correlation. Due to the good performance of our numerical method the $1 / r$ asymptotic behavior can be observed to a large radius without any fitting procedure. According to Niquet et al $\stackrel{43}{=}$ the RPA correlation potential should have a polarization tail of the form $-\alpha_{N}^{\mathrm{RPA}} / 2 r^{4}$, where $\alpha_{N}$ is the static polarizability of the corresponding atom. This effect can, however, only be observed at a considerable distance from the atom, $\approx 20$ bohr radii. An accurate description of the potential at such large distances requires many more basis functions as compared to our standard calculations. Increasing the accuracy in the asymptotic region, a tendency toward such a tail was, however, observed.

Finally, in Fig. 4 the RPA and MP2 correlation potentials of $\mathrm{Mg}$ and $\mathrm{Ar}$ are displayed. The presence of a third s-shell, as well as a second p-shell for Ar, can be observed.

\section{B. Ionization potentials}

Within exact DFT the highest occupied orbital eigenvalue equals the negative of the ionization potential. $\underline{44}$ A further test of the quality of the RPA XC potential is thus a comparison between the eigenenergy of the HOMO and the experimental ionization potential. Results are presented in Table I. In this table we also present the corresponding results obtained from the EXX and from MP2. The latter approximations also give the proper $-1 / r$ tail of the potential which is very important for obtaining a reasonable ionization potential. Therefore, we have not considered it worthwhile to include results from other local potentials like, e.g., those of the LDA or different GGA:s. As can be seen, the ionization potentials of the RPA potential are in very good agreement with experiment and represent a significant improvement on the EXX and also on MP2. Consequently, in this regard, the $v_{\mathrm{xc}}^{\mathrm{RPA}}$ is the best performing potential presently known.

\section{Total energies and role of self consistency}

Using the self-consistent KS orbitals and eigenvalues the total energy was calculated from Eq. (4). The results for different atoms and are presented in TableII. We see that the correlation energy is overestimated by almost a factor of two for the small atoms, leading to a too low total energy. This tendency within the RPA has previously been pointed out by several workers $\stackrel{10,45}{=}$ The MP2 energy functional is seen to perform much better in this regard.

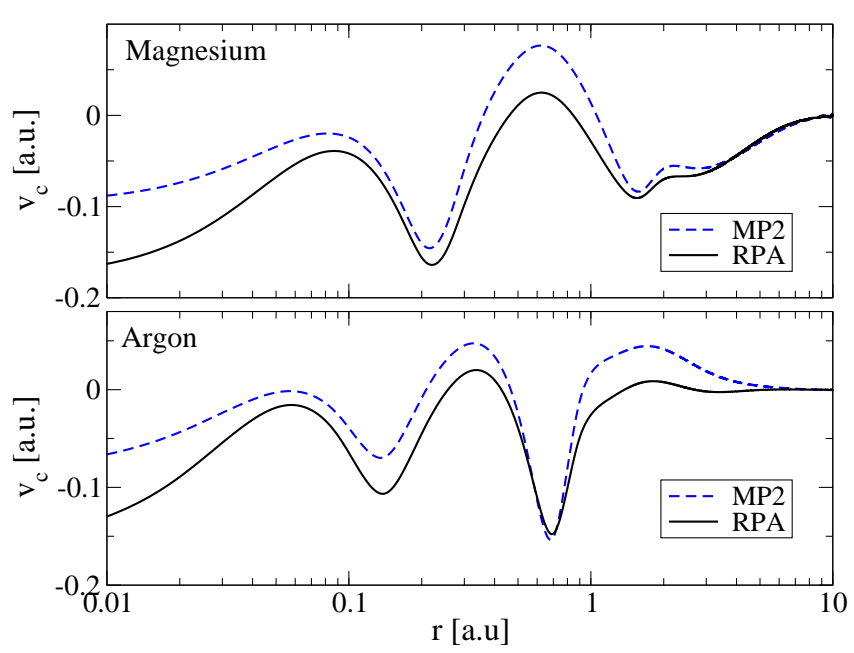

FIG. 4: The RPA and MP2 correlation potentials of Mg and Ar. 
TABLE I: Ionization potentials for some atoms. The RPA potential produces very accurate ionization potentials compared to other potentials with the correct $1 / r$ decay. Values are in Hartrees.

\begin{tabular}{cllll}
\hline \hline Atom & RPA & MP2 & EXX & $\operatorname{Exp}^{b}$ \\
\hline $\mathrm{He}$ & 0.902 & 0.893 & 0.918 & 0.903 \\
$\mathrm{Be}$ & 0.354 & $0.357^{a}$ & 0.309 & 0.343 \\
$\mathrm{Ne}$ & 0.796 & 0.657 & 0.851 & 0.792 \\
$\mathrm{Mg}$ & 0.297 & 0.303 & 0.253 & 0.281 \\
$\mathrm{Ar}$ & 0.590 & 0.560 & 0.591 & 0.579 \\
\hline $\mathrm{MAE}^{c}$ & 0.009 & 0.040 & 0.030 & \\
\hline \hline
\end{tabular}

${ }^{a}$ Calculated using the EXX density.

${ }^{b}$ Experimental data taken from Ref. 34

${ }^{c}$ Mean average error.

TABLE II: Total ground-state energies calculated from the self-consistent density. The RPA energy functional gives too large correlation energies, whereas the MP2 functional performs much better.

\begin{tabular}{crrrr}
\hline \hline Atom & RPA & MP2 & EXX & $\operatorname{Exp}^{a}$ \\
\hline $\mathrm{He}$ & 2.945 & 2.909 & 2.862 & 2.904 \\
$\mathrm{Be}$ & 14.754 & 14.697 & 14.572 & 14.667 \\
$\mathrm{Ne}$ & 129.143 & 129.027 & 128.545 & 128.937 \\
$\mathrm{Mg}$ & 200.296 & 200.129 & 199.612 & 200.059 \\
$\mathrm{Ar}$ & 527.908 & 527.661 & 526.650 & 527.604 \\
\hline \hline
\end{tabular}

${ }^{a}$ Experimental data and MP2 values are taken from Ref. 34.

We have found the Virial Theorem (VT) to be a convenient test of the accuracy of our calculations. Therefore, in Appendix A, this theorem has been proven by us in the case of any conserving density functional approximation. As a matter of fact, in our calculations, the VT is obeyed to within six significant digits. This is a very satisfactory test on the overall accuracy of our calculations. It is important to point out that the VT only holds if the self-consistent orbitals and one-electron eigenvalues are used in the evaluation of the energies.

In order to investigate the variational properties of the total energy of the RPA as a function of the density we have evaluated this functional using orbitals and one-electron eigenvalues also from the LDA, MP2 and the EXX. The results are illustrated in Fig. 5 In all cases we find a higher energy compared to the fully selfconsistent RPA result, in accordance with our previously proven theorem that the RPA density functional has a minimum $\stackrel{33}{3}$ We also studied the variation of the potential with respect to the density used for its evaluation. Only small changes in the potential was observed. Comparing to the results of Jiang and Engel ${ }^{34}$ we conclude that the RPA potential is more stable with respect to variations in the density.

\section{Two-electron excitation energies}

The particle-conserving or two-electron excitation energies can be obtained from the full density response function $\chi$ of the system. In finite systems, there are at least a few such excitation energies below the continuum edge which then show up as poles of $\chi$. Within exact TDDFT these poles are zeros of the expression $\chi_{s}^{-1}-v-f_{\mathrm{xc}}$, where $v$ is the bare Coulomb interaction, $f_{\mathrm{xc}}$ is $\mathrm{XC}$ kernel, and $\chi_{s}$ is the non-interacting KS response function with poles at the differences between the DF eigenvalues. In a one-pole approximation, the twoparticle excitation energies are seen to be the difference between an occupied and an unoccupied DF eigenvalue corrected by some matrix elements with respect to DF orbitals of the Coulomb interaction and the XC kernel, $f_{\mathrm{xc}}$. It has been shown previously by Petersilka et al ${ }^{26}$ that the latter matrix elements have a much smaller influence or effect on the calculated excitation energies than the eigenvalue difference obtained by using different approximations to the XC potential. Of course, the presence of a sum over all poles will affect the actual zeros of the denominator of $\chi$, i.e., the excitation energies. If, however, these zeros are well separated, as is often the case in the discrete part of the spectrum, also this effect is much smaller than the eigenvalue differences produced by different XC potentials. As a consequence, an accurate XC potential is of vital importance for obtaining accurate two-particle excitation energies.

In the Tables $\amalg \square$ the KS eigenvalue differences in RPA are compared to the exact eigenvalue differences obtained from Ref. 25 and to the MP2 and EXX eigenvalue differences. The results show that the mean average error is significantly reduced as compared to the EXX approximation. For He and Be the MP2 approximation also improves the EXX values.

The magnitude of the Hartree contribution and the $f_{\mathrm{xc}}$ contribution to the true excitation energies are presented in Tables VI and VII. We see that the KS eigenvalue differences are already very close to the true excitation

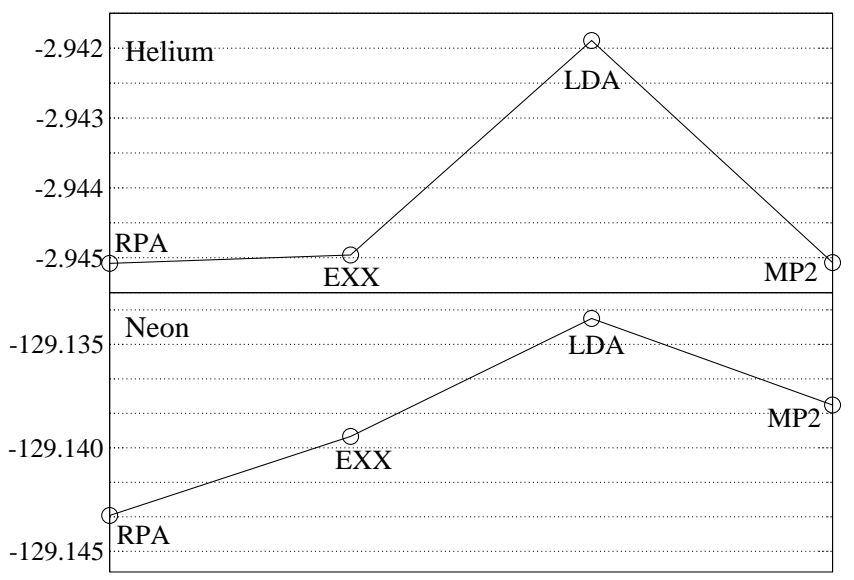

FIG. 5: The total energy for $\mathrm{He}$ and $\mathrm{Ne}$ in the RPA calculated with orbitals and one-electron eigenvalues from the LDA, EXX, MP2 and the RPA. The RPA orbitals are seen to give the lowest energy confirming that the RPA functional has a minimum. 
TABLE III: KS eigenvalue differences for He. The values of the RPA and MP2 improves significantly on the EXX values.

\begin{tabular}{ccccc}
\hline \hline Transition & RPA & MP2 & EXX & Exact $^{a}$ \\
\hline $1 \mathrm{~s} \rightarrow 2 \mathrm{~s}$ & 0.744 & 0.736 & 0.760 & 0.746 \\
$1 \mathrm{~s} \rightarrow 3 \mathrm{~s}$ & 0.844 & 0.836 & 0.861 & 0.839 \\
$1 \mathrm{~s} \rightarrow 2 \mathrm{p}$ & 0.775 & 0.768 & 0.791 & 0.777 \\
$1 \mathrm{~s} \rightarrow 3 \mathrm{p}$ & 0.855 & 0.846 & 0.871 & 0.848 \\
\hline MAE & 0.004 & 0.006 & 0.018 & \\
\hline \hline
\end{tabular}

${ }^{a}$ Taken from Ref. 25.

TABLE IV: KS eigenvalue differences for Be. The errors in the EXX eigenvalue differences are reduces by a factor of two in both the RPA and MP2. Note that the MP2 values are calculated from the EXX density.

\begin{tabular}{ccccc}
\hline \hline Transition & RPA & MP2 & EXX & Exact $^{a}$ \\
\hline $2 \mathrm{~s} \rightarrow 3 \mathrm{~s}$ & 0.254 & 0.253 & 0.214 & 0.244 \\
$2 \mathrm{~s} \rightarrow 2 \mathrm{p}$ & 0.131 & 0.128 & 0.131 & 0.133 \\
$2 \mathrm{~s} \rightarrow 3 \mathrm{p}$ & 0.276 & 0.276 & 0.234 & 0.269 \\
$2 \mathrm{~s} \rightarrow 4 \mathrm{p}$ & 0.332 & 0.330 & 0.297 & 0.305 \\
$2 \mathrm{~s} \rightarrow 3 \mathrm{~d}$ & 0.292 & 0.292 & 0.241 & 0.283 \\
\hline $\mathrm{MAE}$ & 0.011 & 0.011 & 0.023 & \\
\hline \hline
\end{tabular}

${ }^{a}$ Taken from Ref. 25.

TABLE V: KS eigenvalue differences for Ne. The RPA results are almost an order of magnitude better than those of the EXX which are better than those of MP2.

\begin{tabular}{crrrr}
\hline \hline Transition & RPA & MP2 & EXX & Exact $^{a}$ \\
\hline $1 \mathrm{~s} \rightarrow 3 \mathrm{~s}$ & 30.639 & 30.591 & 30.628 & 30.633 \\
$1 \mathrm{~s} \rightarrow 3 \mathrm{p}$ & 30.715 & 30.652 & 30.706 & 30.706 \\
$1 \mathrm{~s} \rightarrow 3 \mathrm{~d}$ & 30.773 & 30.699 & 30.766 & 30.759 \\
$2 \mathrm{~s} \rightarrow 3 \mathrm{~s}$ & 1.462 & 1.336 & 1.526 & 1.469 \\
$2 \mathrm{~s} \rightarrow 3 \mathrm{p}$ & 1.538 & 1.398 & 1.604 & 1.542 \\
$2 \mathrm{~s} \rightarrow 3 \mathrm{~d}$ & 1.595 & 1.444 & 1.664 & 1.595 \\
$2 \mathrm{p} \rightarrow 3 \mathrm{~s}$ & 0.607 & 0.492 & 0.659 & 0.612 \\
$2 \mathrm{p} \rightarrow 3 \mathrm{p}$ & 0.683 & 0.553 & 0.737 & 0.684 \\
$2 \mathrm{p} \rightarrow 3 \mathrm{~d}$ & 0.740 & 0.600 & 0.797 & 0.738 \\
\hline MAE & 0.007 & 0.111 & 0.046 & \\
\hline \hline
\end{tabular}

${ }^{a}$ Taken from Ref. 25 .

energies. Including the Hartree contribution, i.e., evaluating the poles of the RPA response function, drives the KS values further away from the true excitation energies. Including the $f_{\mathrm{xc}}$ part cancels this error and in most cases we come back to a value of the same quality as the KS eigenvalue differences. For some transitions, however, e.g., the $2 \mathrm{~s} \rightarrow 2 \mathrm{p}$ transition in Be, it is necessary to include both the Hartree and the $f_{\mathrm{xc}}$ contributions.

\section{CONCLUSIONS AND DISCUSSION}

In the present work we have calculated that local potential which through a one-particle Schrödinger equation generates the orbitals which minimize the expression for the total energy within the RPA. The systems studied are spherical atoms. We have found that the correlation part of this potential - defined as the total RPA potential minus that corresponding to the EXX - has the effect of softening the shell structure produced by the potential of the EXX. This shell structure consist of rapid oscillations of the XC potential between the atomic shells.

For several spherical atoms, we have compared the spatial dependence of our RPA potential to that of the exact DF potential defined to be the potential which, through the Kohn-Sham procedure, yields the exact electron density of the many-body system. Such exact potentials exist in the cases of the $\mathrm{He}, \mathrm{Be}$, and $\mathrm{Ne}$ atoms. We have found that the RPA potentials are closer to the exact DF potentials than the corresponding local potentials of MP2 theory and much closer than the potentials of the LDA or of any of the GGA:s.

It is relatively difficult to judge the quality of a given local potential from a study of its spatial dependence. Within DFT, the highest occupied eigenvalue ought to equal the negative of the ionization potential. And we have found that our RPA potentials perform very well in this regard, much better than any traditional potential but also better than the local potential from MP2 theory. Our average error in the obtained ionization potentials are $\sim 0.24 \mathrm{eV}$ compared to $\sim 1.1 \mathrm{eV}$ in the case of MP2 theory and $\sim 0.8 \mathrm{eV}$ within the EXX. We interpret this result as being due to the GWA providing a better description of correlation effects among the more loosely bound valence electrons being ionized as compared to the case of MP2.

Another measure of the quality of the calculated RPA potentials can be obtained from a study of two-electron excitation energies. Within TDDFT, and using a singlepole approximation, these can be obtained as differences between DF eigenvalues corrected by relatively small matrix elements of the bare Coulomb interaction and the $\mathrm{XC}$ kernel. The latter corrections are relatively insensitive to the orbitals used in their evaluation and on the choice of XC kernel. Due to the accurate DF potential of the GWA, i.e., the RPA potential, the corresponding eigenvalues are close to exact DF eigenvalues and the resulting two-electron excitation energies are also very accurate.

In the present work, we have shown that the traditional RPA follows from a special choice of variational expression for the total energy involving the GWA for the functional $\Phi$ from which the electronic self-energy is obtained as a functional derivative with respect to the Green function. This guarantees that the density response function obtained by perturbing the system by an external potential will be conserving meaning, e.g., that it will obey the f-sum rule. Another consequence is the fact that the ground-state energies obey the Virial Theorem which is here proven explicitly for any approximation within TDDFT obtained from the variational approach to MBPT.

The demonstrated high quality of the XC potential within the RPA, particularly with regard to energy differences, induces strong hopes that the density response function obtained by perturbing the system will be very 
TABLE VI: Excitation energies for He. In the first three columns the difference between the poles of the response function, in three different approximations, and the experimental values are presented. Note that the poles of $\chi_{s}$ are just the KS eigenvalue differences. The last column gives the experimental values taken from Ref. 26. Already the KS eigenvalue differences are close to the experimental results.

\begin{tabular}{ccccc}
\hline \hline Transition & $\left(\chi_{s}\right)^{-1}$ & $\left(\chi^{\mathrm{RPA}}\right)^{-1}$ & $\left(\chi^{\mathrm{PGG}}\right)^{-1}$ & Exp. \\
\hline $1 \mathrm{~s} \rightarrow 2 \mathrm{~s}$ & -0.014 & +0.010 & +0.006 & 0.758 \\
$1 \mathrm{~s} \rightarrow 3 \mathrm{~s}$ & +0.002 & +0.013 & +0.008 & 0.843 \\
$1 \mathrm{~s} \rightarrow 2 \mathrm{p}$ & -0.005 & +0.005 & +0.002 & 0.780 \\
$1 \mathrm{~s} \rightarrow 3 \mathrm{p}$ & +0.006 & +0.012 & +0.008 & 0.849 \\
\hline MAE & 0.007 & 0.010 & 0.006 & \\
\hline \hline
\end{tabular}

TABLE VII: Excitation energies for Be. The columns presents the same quantities as in Table VI. The $2 \mathrm{~s} \rightarrow 2 \mathrm{p} \mathrm{KS}$ transition is seen to be responsible for the large MAE in the first column and needs to be corrected by the Hartree and the $f_{\mathrm{xc}}$ term.

\begin{tabular}{ccccc}
\hline \hline Transition & $\left(\chi_{s}\right)^{-1}$ & $\left(\chi^{\mathrm{RPA}}\right)^{-1}$ & $\left(\chi^{\mathrm{PGG}}\right)^{-1}$ & Exp. $^{a}$ \\
\hline $2 \mathrm{~s} \rightarrow 3 \mathrm{~s}$ & +0.005 & +0.017 & +0.010 & 0.249 \\
$2 \mathrm{~s} \rightarrow 4 \mathrm{~s}$ & +0.013 & +0.027 & +0.014 & 0.297 \\
$2 \mathrm{~s} \rightarrow 2 \mathrm{p}$ & -0.063 & +0.024 & -0.002 & 0.194 \\
$2 \mathrm{~s} \rightarrow 3 \mathrm{p}$ & +0.002 & +0.012 & +0.007 & 0.274 \\
\hline MAE & 0.020 & 0.020 & 0.008 & \\
\hline \hline
\end{tabular}

${ }^{a}$ Taken from Ref. 26 .

accurate indeed. But this will be the topic of a future publication.

In the present paper we have introduced a novel way of doing electronic structure calculations based on cubic splines as radial basis functions. The original motivation for the introduction of this somewhat unusual basis set was the desire to circumvent numerical difficulties associated with the known singularities of the Kohn-Sham noninteracting density response function. The latter gives no response to a constant potential (long-wave-length limit) and a very small response to a very rapidly varying potential (limit of short wave length). To judge from the high accuracy of our results the splines appear to be ideally suited to deal with the latter problem.

We also want to stress two more advantages of our numerical technique based on the cubic splines. i) the proven accuracy of the re-expansion of a product of two splines in terms of splines guarantees that the matrices corresponding to two-particle propagators like, e.g., response functions, are of the same sizes as one-particle propagators like, e.g., Green functions or - for that matter - wave functions. This property is a clear advantage over more standard basis sets consisting of, e.g, plane waves or LMTO:s. ii) The secular problem based on splines requires the handling of sparse matrices for which there exist efficient standard computer codes. Our nice experience from using the splines on atomic problems suggests that we ought to implement similar methods also in molecules and solids.

Work to apply the ideas introduced in the present pa- per also to calculate density response functions and physical properties like, e.g., polarizabilities, are in progress.

\section{Acknowledgments}

We are grateful to Prof. Umrigar for allowing us to use unpublished results from their work on exact XC potentials. We are also indebted to Dr Engel for communicating data from their calculations on the MP2 potential. M. Stankovski has contributed to our understanding of splines and Prof. Almbladh and Dr Kurth have made several interesting remarks and suggestions. This work was supported by the European Community Sixth Framework Network of Excellence NANOQUANTA (NMP4CT-2004-500198).

\section{APPENDIX A: THE VIRIAL THEOREM}

We will prove that the Virial Theorem holds for any conserving approximation within DFT generated from the Klein functional. In the full many body case the Virial Theorem has already been proved in a conserving approximation $\underline{\underline{46}}$

Consider the Klein functional as a functional of the density $n$ :

$$
Y_{\mathrm{K}}[n]=-i \Phi\left[G_{s}\right]+T_{s}[n]+\int w n+U_{\mathrm{o}}
$$

Keeping the normalization, the density is scaled with respect to the spatial coordinates,

$$
n^{\lambda}=\lambda^{3} n(\lambda \boldsymbol{r}) .
$$

Due to the stationary property of $Y_{\mathrm{K}}$ we have that

$$
\left(\frac{d Y_{K}\left[n^{\lambda}\right]}{d \lambda}\right)_{l=1}=0 .
$$

Let us see how each term in the Klein functional scales when we scale the density as in Eq. A2). The Hartree term scales linearly $(v=1 / r)$

$$
\begin{aligned}
U_{\mathrm{o}}^{\lambda} & =1 / 2 \int d^{3} r d^{3} r^{\prime} v\left(\boldsymbol{r}-\boldsymbol{r}^{\prime}\right) \lambda^{3} n(\lambda \boldsymbol{r}) \lambda^{3} n\left(\lambda \boldsymbol{r}^{\prime}\right) \\
& =l / 2 \int d^{3} r d^{3} r^{\prime} v\left(\boldsymbol{r}-\boldsymbol{r}^{\prime}\right) n(\boldsymbol{r}) n\left(\boldsymbol{r}^{\prime}\right) \\
& =\lambda U_{\mathrm{o}}
\end{aligned}
$$

so

$$
\left(\frac{d U_{\mathrm{o}}^{l}}{d \lambda}\right)_{l=1}=U_{\mathrm{o}} .
$$

The kinetic energy of independent particles is a sum of the occupied one-particle kinetic energies, and is thus an 
implicit functional of the density. With the density scaled as in Eq. (A2) the orbitals scale like

$$
\varphi^{\lambda}=\lambda^{3 / 2} \varphi(\lambda \boldsymbol{r}) .
$$

Inserting the scaled orbitals in the expression for the kinetic energy we find

$$
\begin{aligned}
T_{s}^{\lambda} & =-\frac{1}{2} \sum_{i}^{\text {occ }} \int d^{3} r \lambda^{3 / 2} \varphi_{i}(\lambda \boldsymbol{r}) \nabla^{2} \lambda^{3 / 2} \varphi_{i}(\lambda \boldsymbol{r}) \\
& =-\frac{1}{2} \sum_{i}^{\text {occ }} \int d^{3} r \lambda^{2} \varphi_{i}(\boldsymbol{r}) \nabla^{2} \varphi_{i}(\boldsymbol{r}) \\
& =\lambda^{2} T_{s},
\end{aligned}
$$

and

$$
\left(\frac{d T_{s}^{l}}{d \lambda}\right)_{l=1}=2 T_{s}
$$

The external potential energy $W$ scales as

$$
\begin{aligned}
W^{\lambda} & =\int d^{3} r \lambda^{3} n(\lambda \boldsymbol{r}) w(\boldsymbol{r}) \\
& =\int d^{3} r n(\boldsymbol{r}) w(\boldsymbol{r} / \lambda) .
\end{aligned}
$$

If the external potential is Coulombic we have

$$
\left(\frac{d W^{l}}{d \lambda}\right)_{l=1}=W
$$

The XC energy $E_{\mathrm{xc}}$ is not an explicit functional of $n$ but of $G_{s}$. To see how $E_{\mathrm{xc}}$ scales we must first determine how $G_{s}$ scales, that is, to find that Green function $G_{s}^{l}$, which corresponds to $n^{l}$ for every $l$. Taking into account that the orbitals scale as in Eq. (A5) we have

$$
-\frac{1}{2} \nabla^{2} \varphi_{k}^{\lambda}(\lambda \mathbf{r})=-\frac{\lambda^{2}}{2} \nabla_{\lambda}^{2} \varphi_{k}^{\lambda}(\lambda \mathbf{r}) .
$$

From the KS equation,

$$
\left\{-\frac{1}{2} \nabla^{2}+V(\mathbf{r})\right\} \varphi_{k}(\mathbf{r})=\varepsilon_{k} \varphi_{k}(\mathbf{r}),
$$

we see that

$$
-\frac{\lambda^{2}}{2} \nabla_{\lambda}^{2} \varphi_{k}^{\lambda}(\lambda \mathbf{r})=-\frac{\lambda^{2}}{2}\left\{\varepsilon_{k}-V(\lambda \mathbf{r})\right\} \varphi_{k}^{\lambda}(\lambda \mathbf{r}) .
$$

Thus, the equation that yields the scaled orbitals is

$$
\left\{-\frac{1}{2} \nabla^{2}+\lambda^{2} V(\lambda \mathbf{r})\right\} \varphi_{k}^{\lambda}(\lambda \mathbf{r})=\lambda^{2} \varepsilon_{k} \varphi_{k}^{\lambda}(\lambda \mathbf{r}) .
$$

Consequently, the Green function scales as

$$
G_{s}^{\lambda}\left(\boldsymbol{r}, \boldsymbol{r}^{\prime}, \omega\right)=\lambda G\left(\lambda \boldsymbol{r}, \lambda \boldsymbol{r}^{\prime}, \omega / \lambda^{2}\right) .
$$

A general $\Phi$-diagram of order $n$ can be written

$$
\Phi_{n}=\frac{1}{2 n} \operatorname{Tr} \Sigma_{n}\left[G_{s}\right] G_{s},
$$

where $\Sigma_{n}$ is a skeleton diagram of order $n$. In order $n$ there are thus $n$ interaction lines, $2 n$ coordinates and $2 n$ propagators. Inserting the scaled Green function we find that $\Phi_{n}$ scales as

$$
\Phi_{n}^{l}=\frac{l^{2-n}}{2 n} \operatorname{Tr} \Sigma_{n}\left[G_{s}^{l}\right] G_{s}^{l} .
$$

A factor of $l^{2 n}$ comes from the number of propagators. The variable substitution gives us a factor of $l^{-6 n}$ and from the interaction lines an additional factor of $l^{n}$ is obtained. There are $n+1 \omega$-integrations giving a factor of $l^{2(n+1)}$. In total we obtain a factor of $l^{2-n}$. Taking the $l$-derivative we obtain

$$
\left(\frac{d \Phi_{n}^{l}}{d \lambda}\right)_{l=1}=(2-n) \Phi .
$$

Summing over $n$ yields

$$
\begin{aligned}
\left(\frac{d \Phi^{l}}{d \lambda}\right)_{l=1} & =\sum_{n}\left(\frac{d \Phi_{n}^{l}}{d \lambda}\right) \\
& =\sum_{n}(2-n) \Phi_{n} \\
& =2 \Phi-\sum_{n} n \Phi_{n} \\
& =2 i E_{\mathrm{xc}}\left[G_{s}\right]-i U_{\mathrm{xc}}\left[G_{s}\right] .
\end{aligned}
$$

where

$$
U_{\mathrm{xc}}\left[G_{s}\right]=-\frac{i}{2} \sum_{n} \operatorname{Tr} \Sigma^{n}\left[G_{s}\right] G_{s} .
$$

Summing all the terms we have proved the Virial Theorem

$$
\begin{aligned}
0 & =2 T_{s}+U_{\mathrm{H}}+W+2 E_{\mathrm{xc}}-U_{\mathrm{xc}} \\
& =2 T_{s}+2 T_{\mathrm{xc}}+U_{\mathrm{H}}+W+U_{\mathrm{xc}},
\end{aligned}
$$

where

$$
T_{\mathrm{xc}} \equiv E_{\mathrm{xc}}-U_{\mathrm{xc}} .
$$


1 G. Onida, L. Reining, and A. Rubio, Rev. Mod. Phys. 74, 601 (2002).

2 S. Botti, F. Sottile, N. Vast, V. Olevano, L. Reining, H.C. Weissker, A. Rubio, G. Onida, R. D. Sole, and R. W. Godby, Phys. Rev. B 69, 155112 (2004).

3 Y.-O. Kim and A. Görling, Phys. Rev. B 66, 035114 (2002).

4 M. Petersilka, U. J. Gossmann, and E. K. U. Gross, in Electronic Density Functional Theory: Recent Progress and New Directions, edited by J. F. Dobson, G. Vignale, and M. P. Das (Plenum, New York, 1998), p. 177.

5 S. Kurth and U. von Barth, unpublished.

${ }^{6}$ I. V. Tokatly and O. Pankratov, Phys. Rev. Lett. 86, 2078 (2001).

7 F. Bruneval, F. Sottile, V. Olevano, R. D. Sole, and L. Reining, Phys. Rev. Lett. 94, 186402 (2005).

${ }^{8}$ U. von Barth, N. E. Dahlen, R. van Leeuwen, and G. Stefanucci, Phys. Rev. B 72, 235109 (2005).

9 C. O. Almbladh, U. von Barth, and R. van Leeuwen, Int. J. Mod. Phys. B 13, 535 (1999).

10 N. E. Dahlen and U. von Barth, Phys. Rev. B 69, 195102 (2004).

11 L. M. Luttinger and J. C. Ward, Phys. Rev. 118, 1417 (1960).

12 A. Klein, Phys. Rev. 121, 950 (1961).

13 L. Hedin, Phys. Rev. 139, A796 (1965).

14 W. Kohn and L. J. Sham, Phys. Rev. 140, A1133 (1965).

15 R. T. Sharp and G. K. Horton, Phys. Rev. 90, 317 (1953).

16 J. D. Talman and W. F. Shadwick, Phys. Rev. A 14, 36 (1976).

17 U. von Barth, in Many-Body Phenomena at Surfaces, edited by D. Langreth and H. Suhl (Academic Press, Orlando, Florida, 1984).

18 C. O. Almbladh and A. C. Pedroza, Phys. Rev. A 29, 2322 (1984).

19 D. W. Smith, S. Jagannathan, and G. S. Handler, Int. J. Quantum Chem. Symp. 13103 (1979).

20 F. Aryasetiawan and M. J. Stott, Phys. Rev. B 34, 4401 (1986).

${ }^{21}$ F. Aryasetiawan and M. J. Stott, Phys. Rev. B 38, 2974 (1988).

22 R. W. Godby, M. Schlüter, and L. J. Sham, Phys. Rev. B 36, 6497 (1987).

${ }^{23}$ R. W. Godby, M. Schlüter, and L. J. Sham, Phys. Rev. B
37, 10159 (1988).

${ }^{24}$ It should, however, be remembered that their work was based on pseudo potentials thus excluding the possibility of treating the effect of core-valence exchange in a proper way. The numerical consequences of this is still an open question.

25 C. J. Umrigar and X. Gonze, Phys. Rev. A 50, 3827 (1994).

26 M. Petersilka, E. K. U. Gross, and K. Burke, Int. J. Quantum Chem. 80, 534 (2000).

27 G. Baym and L. Kadanoff, Phys. Rev. 124, 286 (1961).

28 G. Baym, Phys. Rev. 127, 1391 (1962).

29 L. J. Sham, Phys. Rev. B 32, 3876 (1985).

30 L. J. Sham and M. Schlüter, Phys. Rev. B 32, 3883 (1985).

31 M. E. Casida, Phys. Rev. A 51, 2005 (1995).

32 E. K. U. Gross and W. Kohn, Phys. Rev. Lett. 55, 2850 (1985)

33 N. E. Dahlen, R. van Leeuwen, and U. von Barth, Phys. Rev. A 73, 012511 (2006).

34 H. Jiang and E. Engel, J. Chem. Phys. 123, 224102 (2005).

35 Y. Niquet, M. Fuchs, and X. Gonze, Int. J. Quantum Chem. 101, 635 (2004).

36 S. Hirata, S. Ivanov, I. Grabowski, R. J. Bartlett, K. Burke, and J. D. Talman, J. Chem. Phys. 115, 1635 (2001).

37 S. Ivanov, S. Hirata, and R. J. Bartlett, J. Chem. Phys. 116, 1269 (2002).

38 E. Engel, H. Jiang, and A. F. Bonetti, Phys. Rev. A 72, 052503 (2005).

39 R. H. Bartels, J. C. Beatty, and B. A. Barsky, Hermite and Cubic Spline Interpolation (Morgan Kaufmann, San Francisco, 1998).

40 F. Herman and S. Skillman, Atomic Structure Calculations (Prentice-Hall, Englewood Cliffs, New Jersey, 1963).

41 M. Stankovski, Master's thesis, Lund University (2002).

42 F. Aryasetiawan and O. Gunnarsson, Phys. Rev. B 49, 16214 (1994).

43 Y. Niquet, M. Fuchs, and X. Gonze, J. Chem. Phys. 118, 9504 (2003).

44 C. O. Almbladh and U. von Barth, Phys. Rev. B 31, 3231 (1985).

45 F. Furche, Phys. Rev. B 64, 195120 (2001).

46 N. E. Dahlen and R. van Leeuwen, J. Chem. Phys. 122, 164102 (2005). 